\title{
MOLUSCOS RECIENTES DEL CANAL BEAGLE, TIERRA DEL FUEGO: UN ANÁLISIS CUALITATIVO Y CUANTITATIVO DE LOS ENSAMBLES DE VALVAS FÓSILES Y ACTUALES
}

\author{
RECENT MOLLUSKS FROM THE BEAGLE CHANNEL, TIERRA \\ DEL FUEGO: A QUALITATIVE AND QUANTITATIVE ANALYSIS OF \\ BOTH FOSSIL AND MODERN SHELL ASSEMBLAGES.
}

Sandra Gordillo ${ }^{1,2}$, María Sol Bayer ${ }^{1,2} \&$ Julieta Martinelli ${ }^{2}$

\begin{abstract}
In the Isla Grande of Tierra del Fuego, the marine Holocene (ca. 8.000 years BP) is represented by littoral deposits, mostly up to $10 \mathrm{~m}$ a.s.l. raised marine terraces, extending along the modern coast, and characterized by the presence of mollusk shells, mainly bivalves and gastropods. In this work, which is focused on Holocene mollusk assemblages from the Beagle channel, a qualitative and quantitative analysis of both fossil and modern shell assemblages, respectively collected from raised marine deposits and their adjacent beaches, was performed. For each assemblage, species richness, abundance and various taphonomic attributes were analyzed. The results indicate that the differences between assemblages do not correlate with climate changes that occurred during the Mid-Late Holocene, but reflect local differences among the sites taking into account the habitat heterogeneity in the Beagle channel.
\end{abstract}

Key words: marine mollusks, Holocene, Tierra del Fuego.

1 Centro de Investigaciones en Ciencias de la Tierra, Consejo Nacional de Investigaciones Científicas y Técnicas (CICTERRA, CONICET).

2 Centro de Investigaciones Paleobiológicas (CIPAL), Facultad de Ciencias Exactas, Físicas y Naturales, Universidad Nacional de Córdoba. Av. Vélez Sársfield 299 X5000JJC Córdoba, Argentina. sgordillo@efn.uncor.edu 


\section{RESUMEN}

En la isla Grande de Tierra del Fuego, el Holoceno marino (ca. últimos 8.000 años AP) está representado por depósitos litorales, en su mayoría terrazas elevadas hasta $10 \mathrm{~m}$ s.n.m., que se extienden paralelas a la costa actual, y se caracterizan por la presencia de valvas de moluscos, principalmente bivalvos y gasterópodos. En este trabajo, centrado en las asociaciones de moluscos del Holoceno del canal Beagle, se realizó un análisis cualitativo y cuantitativo de los ensambles de valvas fósiles y actuales provenientes, respectivamente, de depósitos marinos elevados y playas adyacentes. Para cada asociación se determinó la riqueza de especies, su abundancia y se analizaron distintos atributos tafonómicos. Los resultados indican que las variaciones entre ensambles no se correlacionan con los cambios climáticos acontecidos durante el Holoceno Medio-Tardío, sino que reflejan diferencias locales entre los sitios considerados dada la heterogeneidad de hábitats en el canal Beagle.

Palabras clave: moluscos marinos, Holoceno, Tierra del Fuego.

\section{INTRODUCCIÓN}

El canal Beagle se encuentra ubicado en el extremo sur de Sudamérica y con una dirección E-O conecta los océanos Atlántico y Pacífico (Fig. 1). La costa norte corresponde a la Isla Grande de Tierra del Fuego, mientras que la costa sur a las islas Hoste y Navarino, separadas por el Canal Murray. La geomorfología actual de este valle tectónico ha sido modelada por los efectos de sucesivas glaciaciones y eventos marinos (Rabassa et al. 2000; Bujalesky 1997) ocurridos durante el período Cuaternario. Esta historia geológica reciente ha provocado además cambios notables en las masas de agua y su circulación, que influenciaron notablemente en la distribución de su biota y la configuración de sus comunidades marinas bentónicas.

En Tierra del Fuego, los cambios del nivel del mar durante el Holoceno, y en menor medida los del Pleistoceno, han quedado registrados por depósitos litorales, en su mayoría terrazas elevadas que se extienden paralelas a la costa actual, y que se caracterizan por la presencia de valvas de moluscos, principalmente bivalvos y gasterópodos (Gordillo \& Cárdenas, 2008; Gordillo 2009), y que constituyen el interés de nuestra investigación.

En el canal Beagle, la última glaciación del Pleistoceno habría tenido lugar hace 24.000 años, y posteriormente hubo un período de retracción gradual de los hielos, hasta su retroceso definitivo producido hacia los 10.000 años A.P. (Rabassa et al. 2000, McCulloch et al. 2005, Kaplan et al. 2008). Al inicio del Holoceno, el canal Beagle, ya transformado en un lago proglacial, siguió alimentándose de los glaciares aún presentes en la cordillera Darwin hasta que alrededor de los 8.000 años A.P. se transformó en un canal marino por el ingreso de aguas procedentes de los océanos circundantes, probablemente a través del Canal Murray (Rabassa et al. 1996, 2000). En ese momento hicieron su aparición en el canal un grupo pionero de taxones euritópicos conformado principalmente por Mytilus chilensis y Mulinia edulis, y que representan los primeros moluscos de este nuevo ambiente marino postglaciar (Gordillo \& Cárdenas 2008, Rabassa et al. 2009).

Ya en el Holoceno Medio, y alrededor de los 6.000 años A.P. el mar alcanzó su máximo nivel (Rabassa et al. 1986, 2000, Gordillo et al. 1992). Respecto a los cambios climáticos acontecidos en esta región en el período que abarca los últimos 6.000 años, otros autores han reconstruido una curva de paleotemperaturas para el canal Beagle en base al análisis de isótopos en valvas de moluscos procedentes de sitios arqueológicos (Fig 2.; Obelic et al. 1998, Strelin et al. 2008). El inicio de la curva se relaciona con un período relativamente más frío que el actual (ca. 6.000-5.000 años AP), seguido de un incremento de la temperatura que alcanzó un máximo en el intervalo de 4.500-4.000 años, en coincidencia con un período de mejoramiento climático a nivel global denominado Hipsithermal. Posteriormente, la temperatura habría descendido nuevamente registrándose varias fluctuaciones menores hasta la Pequeña Edad del Hielo (ca. 400 años $\mathrm{AP}$ ), que fue otro período con temperaturas menores a la actual. 

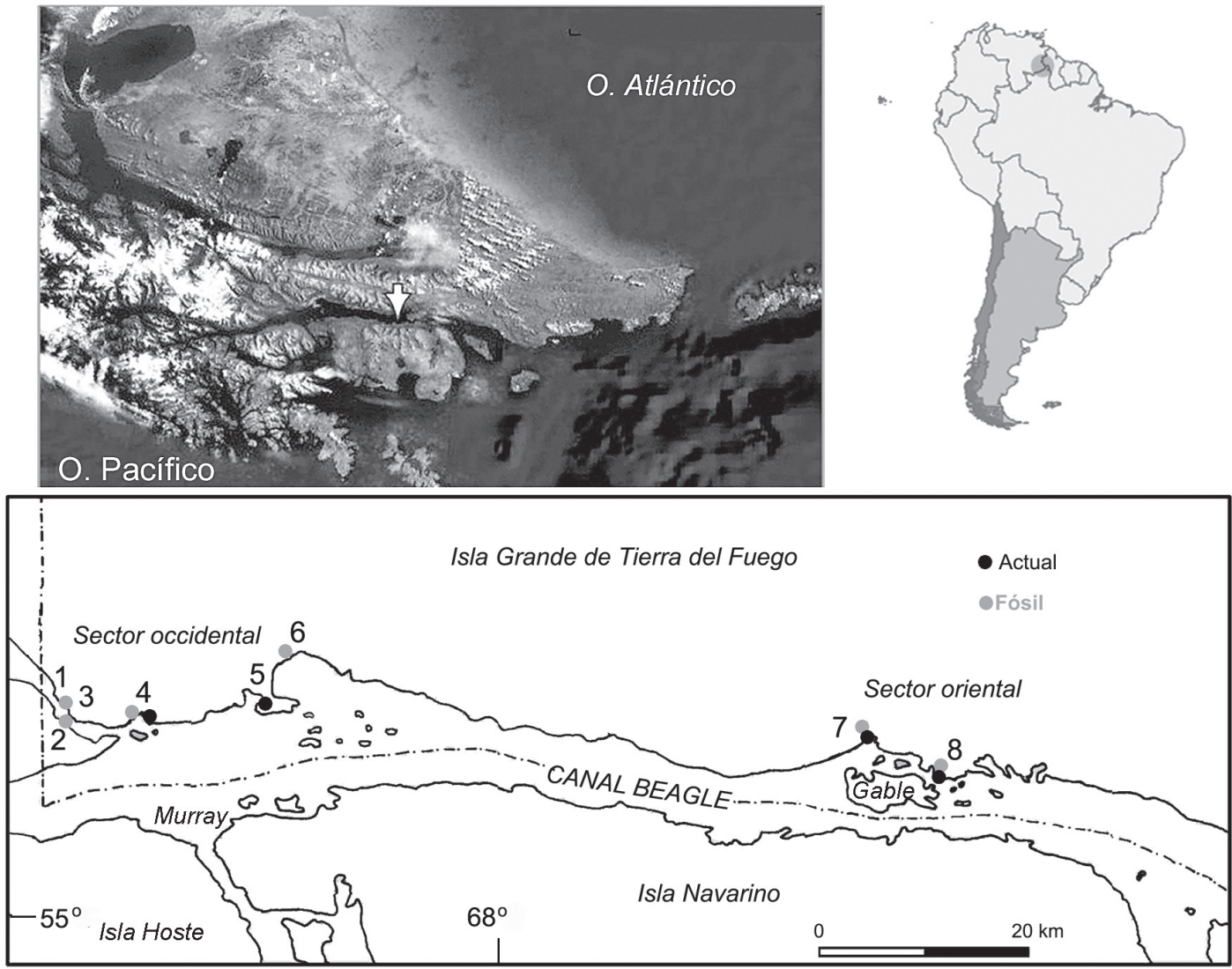

Fig. 1. Mapa de ubicación del Canal Beagle en el extremo sur de Sudamérica y localidades muestreadas. En el sector occidental: 1. Lago Roca. 2. Arch. Cormoranes. 3. Alakush. 4. Bahía Ensenada. 5. Bahía Golondrina. 6. Ushuaia En el sector oriental: 7. Bahía Brown. 8. Estancia Harberton.

En este contexto, y como una contribución al estudio de los cambios climáticos del Cuaternario, resulta importante evaluar si los moluscos marinos del litoral somero de esta región, a nivel comunitario reflejan los cambios ambientales ocurridos en el Holoceno Medio-Tardío. Trabajos previos en el área denotan mayor número de especies hacia los 6.000 años A.P. respecto a los 8.000 años, y mayor diversificación de la fauna de moluscos, especialmente micromoluscos, hacia los 4.500-4.000 años A.P. (Gordillo et al. 2005).

Este trabajo, centrado en las asociaciones de moluscos recientes del canal Beagle, se basa en un análisis cualitativo y cuantitativo de los ensambles de valvas fósiles (Holoceno Medio-Tardío) y actuales provenientes, respectivamente, de depósitos marinos elevados y playas adyacentes.
Características físicas y terrazas marinas del Canal Beagle

El canal Beagle tiene una extensión de 300 $\mathrm{km}$ y un ancho medio de $5 \mathrm{~km}$. Las características hidrológicas, morfológicas y sedimentológicas son muy variables a lo largo del canal (Colizza 1991, Isla et al. 1999, Bujalesky et al. 2004). En proximidades de isla Gable (a la altura del sector oriental de la Fig.1) se produce el encuentro de dos ondas de marea que provienen, una del Atlántico, y la otra del Pacífico. En ese sector, las profundidades oscilan entre 40-50 metros y los fondos son rocosos con guijarros y bioclastos. Por otro lado, $50 \mathrm{~km}$ al este de isla Gable (a la altura del sector occidental de la Fig. 1) la onda de marea proviene del Pacífico, y se registran profundidades mayores a 100 metros, con predominio de fondos con fracción fina limo-arcilloso 
a arenoso. La amplitud media de marea en el canal es de $1.1 \mathrm{~m}$ (Servicio de Hidrografía Naval, 1981). Una característica común de toda la costa norte del canal es la alternancia de pendientes rocosas con entrantes de grava y/o arena que conforman ambientes hidrodinámicos restringidos.

En el canal Beagle, el primero en reconocer la existencia de terrazas de origen marino fue Halle (1910). Luego Urien (1968) reafirmó su presencia y describió 3 niveles marinos en proximidades de la ciudad de Ushuaia. Posteriormente, otros autores también reconocieron varios niveles en otras localidades a lo largo del canal (Porter et al. 1994; Rabassa et al. 1986; Gordillo et al. 1992, 1993). Las máximas alturas del mar (8-10 m s.n.m.) han sido registradas ca. 6.000 años A.P., decreciendo luego, progresivamente, hasta alcanzar el nivel actual (Rabassa et al. 2000). Sin embargo, estas terrazas se encuentran a diferentes alturas a lo largo del canal, debido a un ascenso tectónico diferencial (Bujalesky 1997) que ha continuado ininterrumpidamente hasta la actualidad.

\section{MATERIAL Y MÉTODOS}

Para la realización de este estudio se recolectaron valvas de moluscos actuales $(\mathrm{N}=1257)$ y fósiles ( $\mathrm{N}=479)$ de diferentes localidades ubicadas sobre la costa norte del mencionado canal (Fig. 1; Tabla 1).

Las valvas de moluscos actuales fueron colectadas utilizando un muestreador de $1 \mathrm{~m}^{2}$ o cuadrata, mientras que el material paleontológico se obtuvo a partir de muestras volumétricas de sedimento, que posteriormente fueron tamizadas. Todos los especímenes que componen la macrofauna de moluscos fueron identificados a la menor categoría taxonómica posible. Se determinó el número de especies encontradas, la abundancia relativa, la uniformidad y la diversidad específica. Los parámetros ecológicos de diversidad de cada ensamble fueron calculados utilizando el programa PAST (Hammer et al. 2005). Las diferencias y similitudes entre los ensambles se establecieron mediante análisis multivariado aplicando una transformación de raíz cuadrada a los datos y utilizando la distancia de similitud de Bray-Curtis.

Para evaluar la preservación de los conjuntos faunísticos se analizó la variación tafonómica de los dos bivalvos mejor representados y que además tienen diferente modo de vida. Estas dos especies son: Mytilus chilensis que es epifaunal y vive adherido al sustrato por los filamentos del biso; y Tawera gayi que es infaunal, y de hábito excavador. Los atributos tafonómicos considerados fueron la articulación/ desarticulación, la fragmentación y el desgaste de las valvas, que se explican brevemente a continuación. La articulación se refiere a la presencia de las dos valvas (derecha e izquierda) unidas. Cuando están desarticuladas, se calcula la relación entre valvas derechas e izquierdas que permite estimar el grado de transporte de cada ensamble desde la zona de producción hasta la zona de sedimentación. La fragmentación se asocia a la ruptura, y en general tiene lugar en ambientes de alta energía (ej. playas y paleoplayas) por el impacto con otras valvas, rocas, o por el oleaje (Parsons \& Brett 1991; Zuschin et al. 2003), aunque puede asociarse a otras causas

TABLA 1, Coordenadas, altura (s,n,m,) y edad de los sitios estudiados,

\begin{tabular}{|c|c|c|c|}
\hline LOCALIDADES & $\operatorname{ALTURA}(\mathrm{s}, \mathrm{n}, \mathrm{m})$, & Coordenadas & 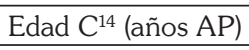 \\
\hline Lago Roca (LR) & $6,4 \mathrm{~m}$ & $54^{\circ} 48^{\prime} \mathrm{S}, 68^{\circ} 36^{\prime} \mathrm{O}$ & $5920+/-90^{(2)}$ \\
\hline Arch. Cormoranes (AC) & $3,0 \mathrm{~m}$ & $54^{\circ} 51^{\prime} \mathrm{S}, 68^{\circ} 35^{\prime} \mathrm{O}$ & $4425+/-55^{(2)}$ \\
\hline Alakush $(\mathrm{AK})$ & $5,0 \mathrm{~m}$ & $54^{\circ} 50^{\prime} \mathrm{S}, 68^{\circ} 34^{\prime} \mathrm{O}$ & $4440+/-120^{(1)}$ \\
\hline Bahía Ensenada (BE) & $2,2 \mathrm{~m}$ & $54^{\circ} 51^{\prime} \mathrm{S}, 68^{\circ} 30^{\prime} \mathrm{O}$ & $2120+/-45^{(4)}$ \\
\hline Ushuaia (US) & $8,0 \mathrm{~m}$ & $54^{\circ} 00^{\prime} \mathrm{S}, 68^{\circ} 16^{\prime} \mathrm{O}$ & $5160+/-130^{(3)}$ \\
\hline Bahía Brown (BB) & $2,6 \mathrm{~m}$ & $54^{\circ} 53^{\prime} \mathrm{S}, 67^{\circ} 31^{\prime} \mathrm{O}$ & $985+/-135^{(4)}$ \\
\hline Ea. Harberton (HA) & $2,3 \mathrm{~m}$ & $54^{\circ} 53^{\prime} \mathrm{S}, 67^{\circ} 24^{\prime} \mathrm{O}$ & $2770+/-50^{(4)}$ \\
\hline Bahía Ensenada (BE) & $0,5 \mathrm{~m}$ & $54^{\circ} 51^{\prime} \mathrm{S}, 68^{\circ} 30^{\prime} \mathrm{O}$ & Actual \\
\hline Bahía Golondrina (BG) & $0,5 \mathrm{~m}$ & $54^{\circ} 49^{\prime} \mathrm{S}, 68^{\circ} 19^{\prime} \mathrm{O}$ & Actual \\
\hline Bahía Brown (BB) & $0,5 \mathrm{~m}$ & $54^{\circ} 53^{\prime} \mathrm{S}, 67^{\circ} 31^{\prime} \mathrm{O}$ & Actual \\
\hline Ea. Harberton (HA) & $0,5 \mathrm{~m}$ & $54^{\circ} 53^{\prime} \mathrm{S}, 67^{\circ} 24^{\prime} \mathrm{O}$ & Actual \\
\hline
\end{tabular}

Edades Carbono 14: (1) Figuerero \& Mengoni 1980; (2) Rabassa et al, 1986; (3) Gordillo 1990; Gordillo et al, 1992 , 
como cambios bruscos de temperatura y congelamiento. Para medir la fragmentación, las valvas se clasificaron en fragmentadas y no fragmentadas. El desgaste en este caso se refiere a la pérdida de ornamentación externa, ya sea asociada a razones mecánicas o agentes abrasivos, o químico-mecánicas o corrasión (Brett \& Baird 1986; Parsons \& Brett 1991). Otros dos atributos, la bioerosión e incrustación fueron evaluados como presencia o ausencia en todos los ejemplares del conjunto faunístico. La bioerosión se evaluó en base a la alteración en las valvas producidas por gasterópodos perforadores de valvas, que se alimentan de bivalvos y otros moluscos. Estas marcas aparecen como orificios circulares en las valvas de moluscos y fueron identificadas a partir de trabajos previos (Gordillo 1994; Gordillo \& Amuchástegui 1998). Finalmente se consideró la incrustación en referencia a los epibiontes o aquellos organismos que usan las valvas de moluscos como sustrato. Para el análisis tafonómico las distintas localidades se reagruparon en cuatro grupos (Fósil Oeste, Fósil Este, Actual Oeste y Actual Este) a los fines de evaluar las diferencias y similitudes entre el sector oriental y el sector occidental, y entre moluscos fósiles y actuales.

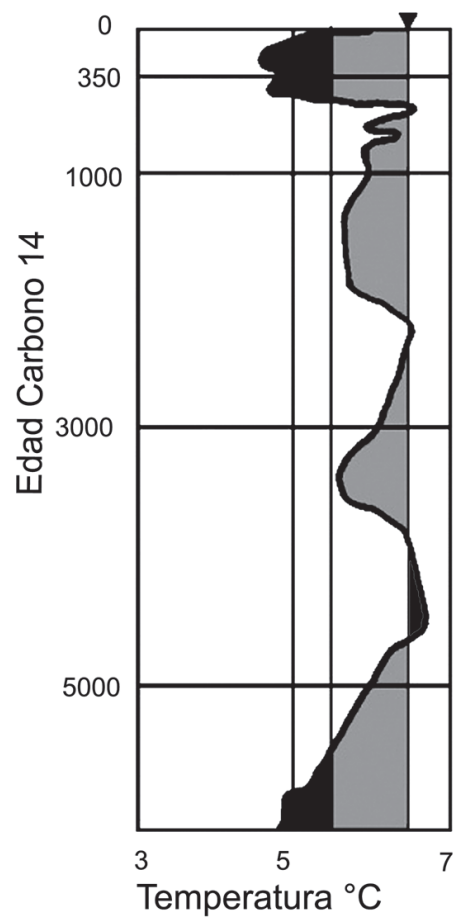

Fig. 2. Curva de paleotemperaturas para el Canal Beagle obtenida en base a un análisis isotópico realizado a partir de conchillas de Mytilus de sitios arqueológicos (Obelic et al. 1998; gráfico modificado de Strelin et al. 2008).

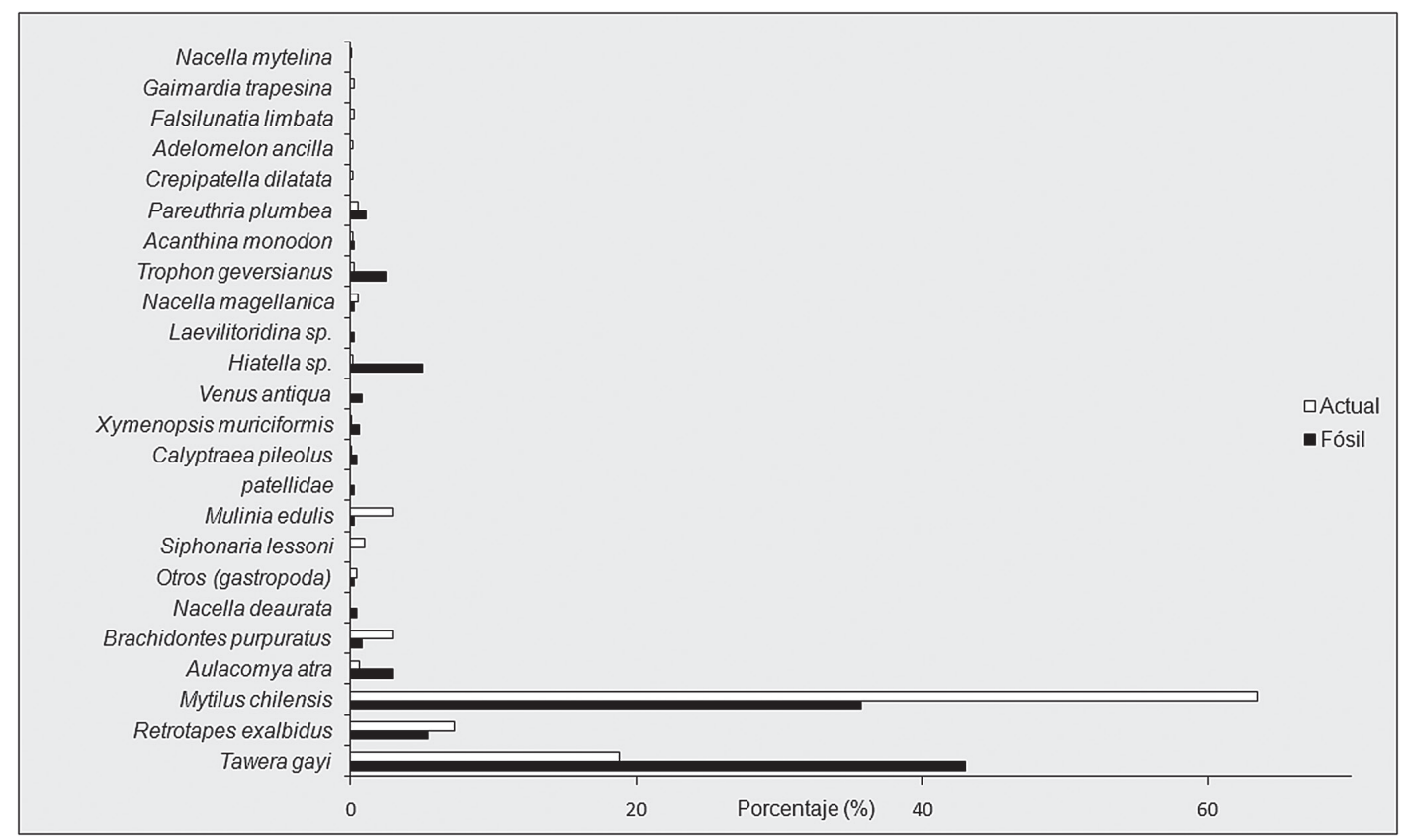

Fig. 3. Abundancia relativa comparativa de ensambles fósiles y actuales. 


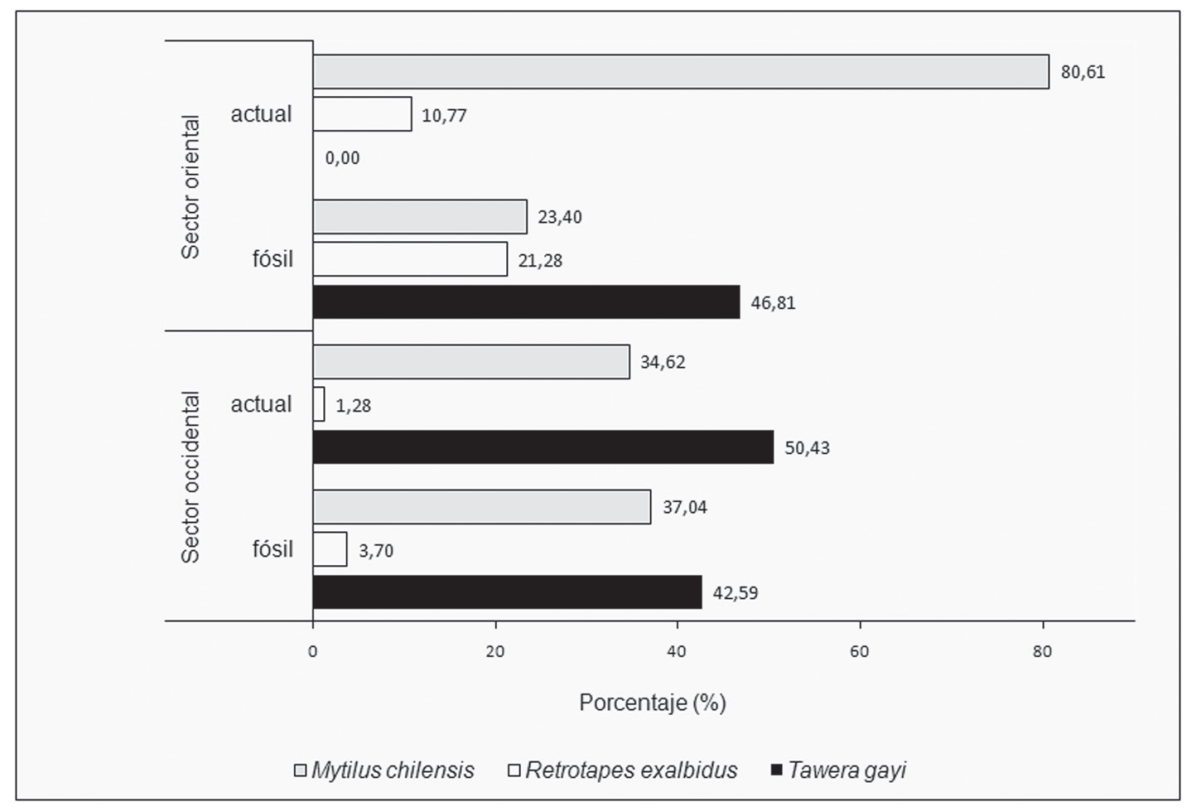

Fig. 4. Abundancia relativa comparativa entre el sector occidental y el sector oriental de tres especies dominantes en los ensambles fósiles y actuales.

\section{RESULTADOS}

\section{Sistemática y abundancia}

Tanto los ensambles actuales como fósiles exhiben una buena preservación, y son tafonómicamente comparables. El 80-90\% de estos ensambles estuvo representado por 3 especies: Mytilus chilensis, Tawera gayi y Retrotapes exalbidus, y el porcentaje restante por más de 20 taxones aprox. (Figs. 3 y 4).

Articulación, relación valvas derechas e izquierdas, fragmentación y abrasión

La articulación solamente se evaluó en ensambles actuales del sector este donde una proporción de ejemplares de Mytilus chilensis estaban articulados. Sin embargo, las dos especies analizadas presentaron similar proporción de valvas derechas e izquierdas tanto en ensambles actuales como fósiles (Fig. 5A). Respecto a la fragmentación, todas las valvas de Mytilus chilensis fósiles, y las valvas actuales de oeste, mostraron un 100\% de fragmentación, mientras que en los ensambles actuales del sector oriental, ese porcentaje fue considerablemente menor (Fig. 5B). En cuanto a las valvas de Tawera gayi fósiles, tanto las del este como las del oeste presentaron una fragmentación del orden del 40\%, mientras que en los ensambles actuales los valores de fragmentación resultaron menores, con información sobre Tawera gayi sólo para el sector oeste, con casi la totalidad de valvas enteras (97,5\%; Fig. 5B). También para el sector este, las valvas de otro Veneridae bien representado (Retrotapes exalbidus), presentaron muy bajo porcentaje de valvas fragmentadas.

Otro atributo considerado fue el desgaste sufrido en la superficie externa de las valvas. Comparativamente, las valvas de Mytilus chilensis presentaron menor desgaste que las valvas de Tawera gayi, llegando incluso a ser nulo en los ensambles actuales del este (Fig. 5C).

\section{Bioerosión e incrustación}

Con respecto a las marcas producidas por otros organismos o signos de interacciones biológicas, en el sector este se observó el mismo porcentaje de valvas actuales y fósiles con incrustaciones (20\%), mientras que en el oeste el valor obtenido fue ligeramente menor (14\%). En relación a la bioerosión, el 7\% de las valvas del sector este (tanto fósiles como actuales) presentaron signos de depredación; 
A

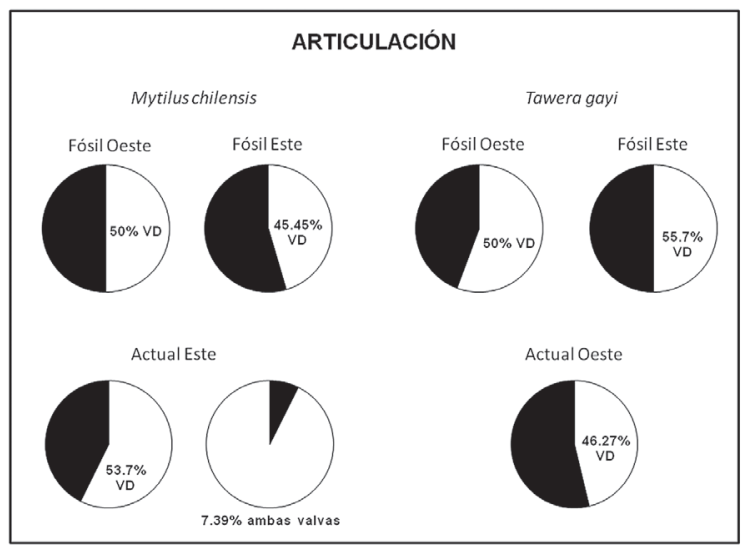

C

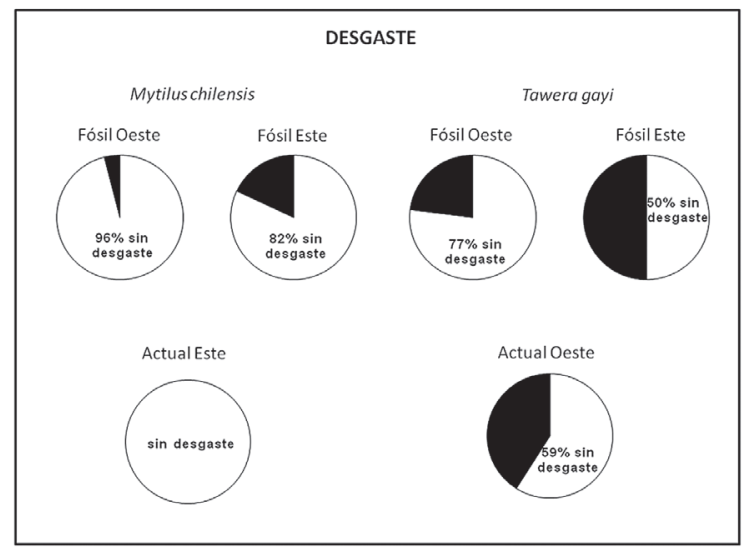

B

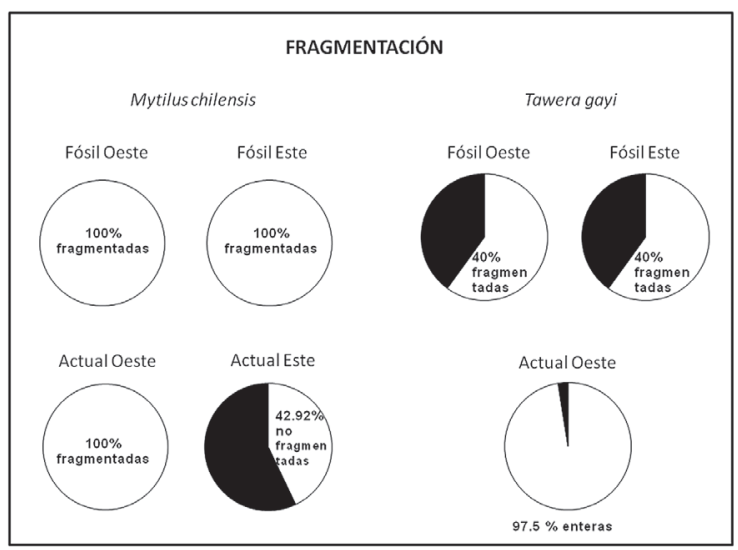

D

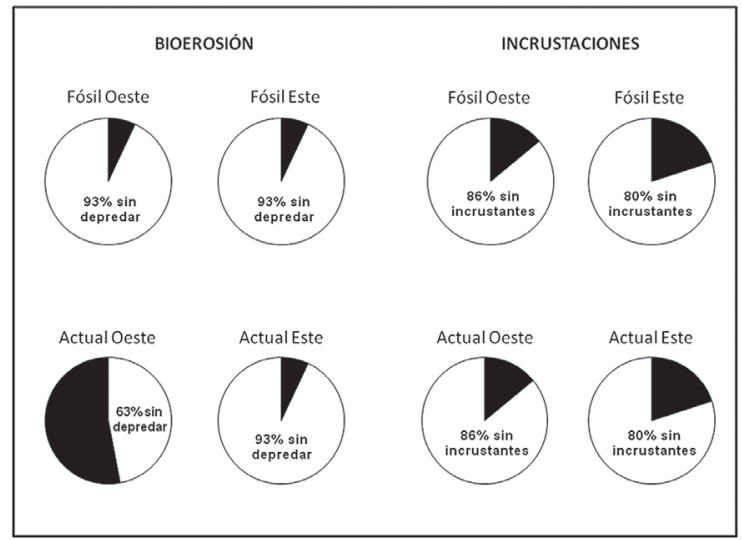

Fig. 5. Proporción de los atributos tafonómicos en un bivalvo infaunal (Tawera gayi) y otro epifaunal (Mytilus chilensis) dominantes en el Holoceno del Canal Beagle. A. Porcentajes de articulación y relación de valvas derechas (VD) e izquierdas. B. Porcentajes de fragmentación. Valvas enteras (negro). C.

Porcentajes del desgaste sobre la superficie externa de la valva. D. Porcentajes de bioerosión producidas por gasterópodos perforadores e incrustación por organismos que usan las valvas como sustrato.

mientras que para el sector oeste los porcentajes fueron diferentes: aprox. 7\% en ejemplares fósiles, y $47 \%$ en valvas actuales (Figura 5D). Este último porcentaje está dado principalmente por valvas de Tawera gayi perforadas procedentes de la playa actual de Bahía Golondrina. Entre las especies que presentaron signos de bioerosión se mencionan Retrotapes exalbidus, Mulinia edulis, Tawera gayi, Mytilus chilensis y Hiatella sp. (Figura 6). Estas marcas de bioerosión fueron analizadas en trabajos previos (Gordillo 1994; Gordillo \& Amuchástegui 1998) y, por su morfología, fueron atribuidas a gasterópodos murícidos (i.e. Trophon geversianus y Xymenopsis muriciformis) que se alimentan de estos bivalvos.
Riqueza de especies, equidad, dominancia y similitud

A lo largo del canal Beagle, los ensambles de moluscos fósiles presentaron índices de diversidad variable, mientras que los ensambles actuales presentaron diversidad decreciente desde el este hacia el oeste, y los mayores valores de diversidad tanto de ensambles fósiles como actuales correspondieron a la localidad de Bahía Ensenada. Respecto a la dominancia ecológica, los mayores valores se registraron en el sector oriental, y la uniformidad se evidenció en los ensambles fósiles tanto del este como del oeste (Fig. 7). 


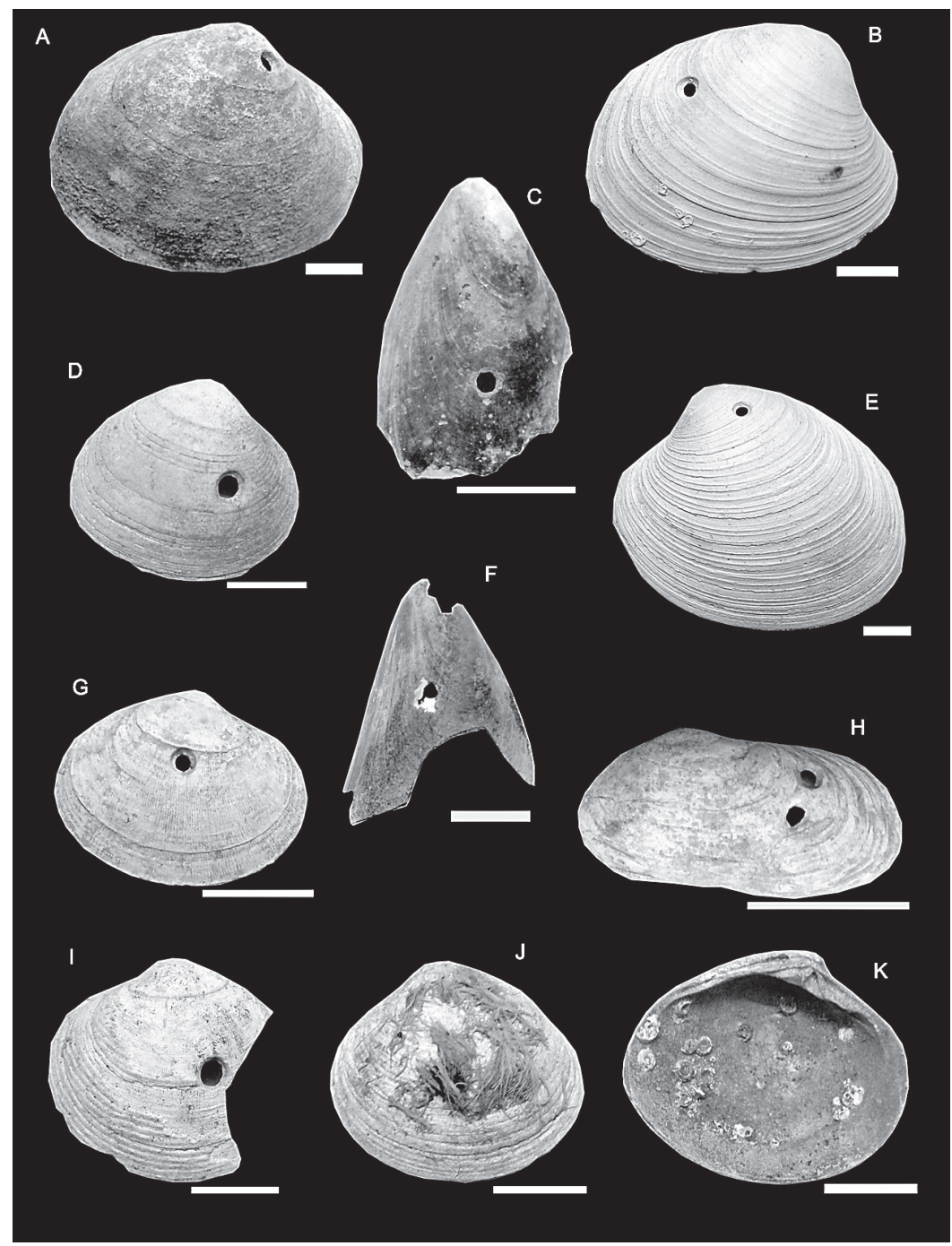

Fig. 6. Valvas con signos de fragmentación, desgaste, bioerosión e incrustación. Fragmentación (C, F, I). Desgaste (G, I). Bioerosión (A-I). Incrustación (J, K). Especies: Retrotapes exalbidus (B, E); Mulinia edulis (A); Tawera gayi (D, G,

I, J, K); Mytilus chilensis (C, F) y Hiatella sp. (H). Valvas actuales (A, B, D, E, F, J, K). Valvas fósiles (C, G, H, I).

Los resultados del análisis de similitud entre las localidades se presentan en el dendrograma de la Fig. 8. En ese gráfico se observa un patrón anidado en que los ensambles actuales incluyen a los ensambles fósiles. Esta configuración, a pesar de las razones metodológicas en agrupaciones jerárquicas, podría explicarse como "un gradiente temporal por razones tafonómicas" ya que las muestras actuales incluyen a todas las especies presentes en las muestras fósiles. La localidad de Bahía Brown caracterizada por la presencia de Mulinia edulis fue la única que se diferenció del resto.

\section{DISCUSIÓN Y CONCLUSIONES}

Los ensambles de moluscos analizados en este trabajo son en su mayoría de tipo alóctono (Kidwell \& Bosence, 1991) ya que se originaron en el intermareal y submareal (zona de producción) y fueron transportados hasta el supralitoral (zona de sedimentación). Los atributos tafonómicos permitieron evaluar los procesos energéticos que intervinieron en su formación, concluyendo que las valvas de bivalvos que conforman estos ensambles fueron arrastradas juntas durante eventos de tormenta de alta energía 


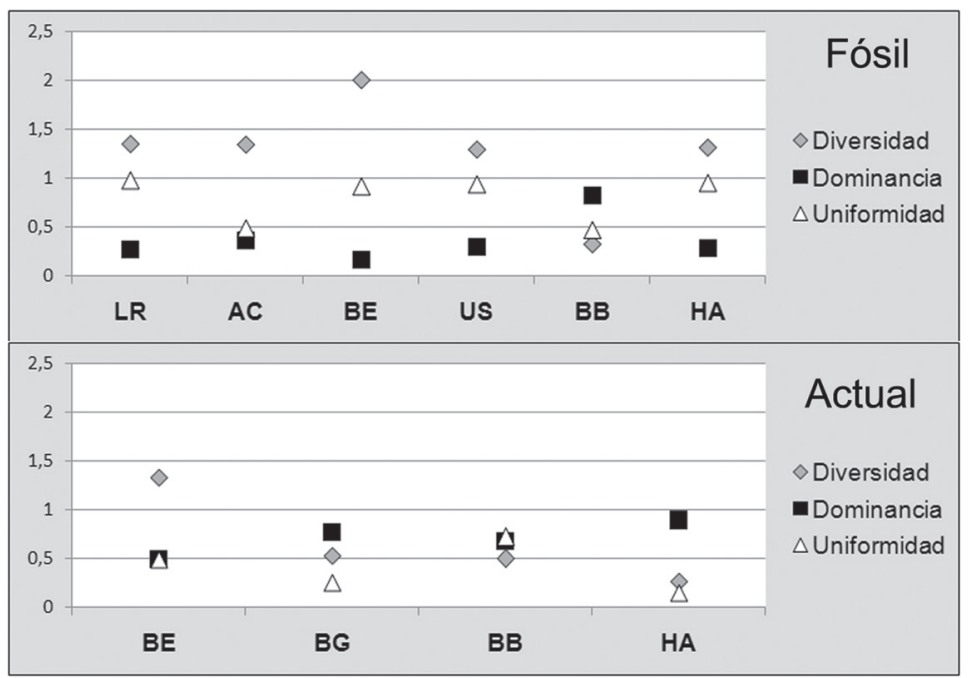

Fig. 7. Valores de los índices de diversidad y de uniformidad de Shannon, y dominancia ecológica de Simpson en ensambles fósiles y actuales. Las iniciales corresponden a las localidades en la Tabla 1.

$y$, si estaban separadas, no sufrieron desplazamiento lateral diferencial. Los signos de desgaste y fragmentación evidenciados fueron variables en las dos especies analizadas, siendo mayor la fragmentación y menor el desgaste en Mytilus chilensis que en Tawera gayi. Estas diferencias resultan de las diferencias en la microestructura y grosor de estas valvas (características intrínsicas) que responden de manera diferencial a los efectos del ambiente, pero también se asocian a las variaciones en las condiciones hidrodinámicas durante el transporte hacia los diferentes sitios donde fueron halladas, sumados a las características físico-químicas de los ambientes de depositación. Por ejemplo, las valvas de Tawera gayi fósiles procedentes del sitio Arch. Cormoranes, que actualmente es un ambiente dulceacuícola, presentan la superficie desgastada por corrosión, con exposición de las capas internas, pero están menos fragmentadas que Mytilus chilensis. Las valvas de Mytilus chilensis en cambio aparecen rotas en la mayoría de las localidades y, aparentemente, siguen un patrón de rotura característico descripto por Zuschin \& Stanton (2001) para Mytilus edulis que consiste en una única fractura (o más) perpendiculares

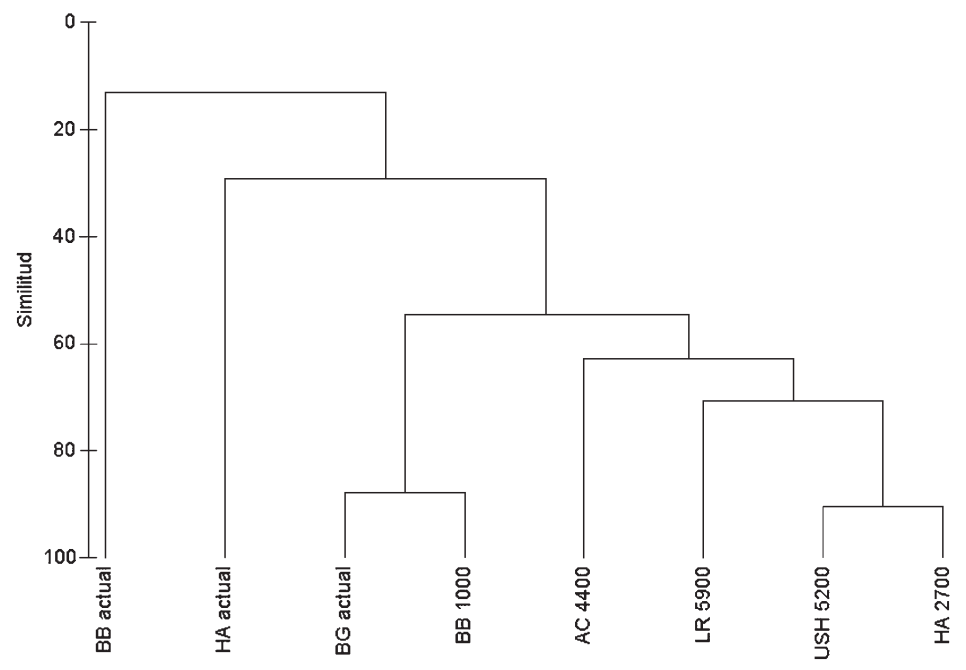

Fig. 8. Dendrograma jerárquico con transformación de raíz cuadrada usando la distancia de similitud de BrayCurtis. Las iniciales corresponden a las localidades en la Tabla 1 y los números a sus edades aproximadas. 
al eje mayor de la valva. Sin embargo este patrón no ha sido aún evaluado en la región.

La depredación de Trophon geversianus, que es un gasterópodo epifaunal, sobre bivalvos infaunales es factible ya que estos bivalvos no siempre viven completamente enterrados, sino que al ser móviles y someros, muchas veces adoptan un modo de vida semi-infaunal, e incluso epifaunal recostados sobre una de sus valvas, estando más expuestos a estos depredadores. Pero además, el alto porcentaje de valvas de Tawera gayi perforadas en los ensambles actuales de bahía Golondrina también podría asociarse a eventos de alta energía, frecuentes en esa región expuesta a los vientos del oeste, que hayan provocado la exhumación de los especímenes de Tawera gayi que vivían enterrados o semi-enterrados en el mesolitoral inferior e infralitoral, quedando más vulnerables a la acción de los gasterópodos perforadores. Sin embargo, otros factores como la disponibilidad de sustrato adecuado, o variaciones en la intensidad de la interacción depredador-presa a nivel local, deberán ser también analizados para nuevas y más ajustadas interpretaciones.

Al comparar los ensambles actuales y fósiles no se observaron cambios composicionales de la macrofauna asociados a las fluctuaciones de temperatura que tuvieron lugar en la región durante el Holoceno Medio y Tardío, lo que se interpreta como una estabilidad de las comunidades de moluscos de aguas someras aún ante ligeros cambios de las condiciones climáticas. Sin embargo, las principales variaciones entre ensambles responden a razones ecológicas asociadas a la heterogeneidad de hábitats en el canal como resultado de su historia geológica reciente. El patrón anidado de los ensambles permite caracterizar una mega-asociación para el canal Beagle con ligeras variaciones espaciotemporales que obedece al desarrollo de diversas comunidades bentónicas locales con predominio de diferentes elementos epifaunales e infaunales, quizás según los tipos de sustrato predominantes en cada sitio.

Se concluye que las diferencias cuali-cuantitativas de los ensambles fósiles y actuales estudiados obedecen, principalmente, a características locales y ambientales de cada sitio, y no reflejan claramente los patrones regionales asociados a las fluctuaciones de la temperatura que tuvieron lugar durante el Holoceno Medio y Tardío.
Teniendo en cuenta que los efectos de las variables ambientales se producen a distintos niveles de la organización biológica, puede suceder que aunque a nivel comunitario ocurra estabilidad, las poblaciones de cada especie respondan de manera distinta a dichos cambios. Por tal motivo, trabajos futuros en el área se complementarán con nuevos análisis isotópicos en valvas de moluscos, y análisis morfométricos y esclerocronológicos a nivel de especie para establecer con mayor claridad las variaciones ambientales y los efectos paleoclimáticos sobre los moluscos marinos en el extremo austral de Sudamérica.

\section{AGRADECIMIENTOS}

Para este trabajo se contó con financiamiento del Consejo Nacional de Investigaciones Científicas y Técnicas (PIP 09-260).

\section{LITERATURA CITADA}

Brett, C. \& G. Baird 1986. Comparative taphonomy: a key to paleoenvironmental interpretation based on fossil preservation. Palaios 1: 207-227.

Bujalesky, G., S. Aliotta \& F. Isla 2004. Facies del subfondo del canal Beagle, Tierra del Fuego. Revista de la Asociación Geológica Argentina 59: 29-37.

Bujalesky, G.G. 2007. Coastal geomorphology and evolution of Tierra del Fuego (Southern Argentina). Geologica Acta 5: 337-362.

Figuerero, M.J. \& G.L. Mengoni 1986. Excavaciones arqueológicas en la Isla El Salmón, Parque Nacional de Tierra del Fuego. Informes de Investigación 4: 1-195.

Colizza, E. 1991. Preliminary report on coastal morphology and sea-bottom sediments of the canales Beagle, Ballenero, Brecknock, Cockburn and Magdalena. Bolletino di Oceanologia Teorica ed Applicata 9: 273-279.

Gordillo, S. 1990. Malacofauna de los niveles marinos holocenos de la Península Ushuaia y alrededores (Canal Beagle, Argentina). En Reunión de campo de Geología del Cuaternario, 3: 24-25. Bahía Blanca, Argentina.

Gordillo, S. 1994. Perforaciones en bivalvos subfósiles y actuales del Canal Beagle, Tierra del Fuego. Ameghiniana 31: 177-185. 
Gordillo S. 2009. Quaternary marine mollusks in Tierra del Fuego: insights from integrated taphonomic and paleoecologic analysis of shell assemblages in raised deposits. Anales Instituto Patagonia, Chile 37: 5.36.

Gordillo S. \& S. Amuchástegui 1998. Estrategias de depredación del gastrópodo perforador Trophon geversianus (Pallas) (Muricoidea: Trophonidae). Malacologia 39: 83-91.

Gordillo S., G. Bujalesky, P.A. Pirazzoli, J.O. Rabassa \& J.F. Saliege 1992. Holocene raised beaches along the northern coast of the Beagle Channel, Tierra del Fuego, Argentina. Palaeogeography, Palaeoclimatology, Palaeoecology 99: 41-54.

Gordillo, S., A.M.J. Coronato \& J.O. Rabassa 1993. Late Quaternary Evolution of a Sub-Antarctic Paleofjord, Tierra-Del-Fuego. Quaternary Science Reviews 12: 889-897.

Gordillo, S., A.M.J. Coronato \& J.O. Rabassa 2005. Quaternary molluscan faunas from the island of Tierra del Fuego after the Last Glacial Maximum. Scientia Marina 69 (Suppl. 2): 337-348.

Gordillo, S. \& J. Cárdenas 2008. Moluscos cuaternarios del extremo austral de América: avances y perspectivas. En: Actas del Primer Simposio Paleontología de Chile: 14-20. Santiago, Chile.

Halle, T.G. 1910. On Quaternary deposits and changes of level in Patagonia and Tierra del Fuego. Bulletin of the Geological Institution of the University of Upsala 9: 93-117.

Hammer, Ø., D.A.T. Harper \& P.D. Ryan 2005. PAST-PAlaeontological Statistics, ver. 1.35. http://folk.uio.no/ohammer/past.

Isla, F., G. Bujalesky \& A. Coronato 1999. Dinámica estuarina del Canal Beagle, Tierra del Fuego. Revista de la Asociación Geológica Argentina 54: 307-318.

Kaplan M.R., C.J. Fogwill, D.E. Sugden, N.R.J. Hulton, P.W. Kubik \& S.P. Freeman 2008. Southern Patagonian glacial chronology for the Last Glacial period and implications for Southern Ocean climate. Quaternary Science Reviews 27: 284-294.

Kidwell, S.M. \& D.W.J. Bosence 1991. Taphonomy and time-averaging of marine shelly faunas. En: Allison, A. \& D.E.G. Briggs (eds.): Ta- phonomy: releasing the data locked in the fossil record, Plenum Press, New York, pp. 115-188.

McCulloch R.D., C.J. Fogwill, D.E. Surgen, M.J. Bentley \& P.W. Kubik 2005. Chronology of the last glaciation in central Strait of Magellan and Bahía Inútil, southernmost South America. Geografiska Annaler 87 A: 289-312.

Obelic, B., A. Álvarez, J. Argullós \& E.L. Piana 1998. Determination of water palaeotemperature in the Beagle Channel (Argentina) during the last 6000 yr through stable isotope composition of Mytilus edulis shells. Quaternary of South America and Antarctic Peninsula: 11: 47-71.

Parsons, K. \& C. Brett 1991. Taphonomic processes and biases in modern marine environments: an actualistic perspective on fossil assemblage preservation. En: Donovan, S. (ed.): The Processes of Fossilization. Columbia University Press, New York, pp. 22-65.

Porter, S., M. Stuiver \& C. Heusser 1984. Holocene sea-level changes along the Strait of Magellan and Beagle channel, South America. Quaternary Research: 22: 59-67.

Rabassa, J., C. Heusser \& R. Stuckenrath 1986. New data on Holocene Sea Transgression in the Beagle channel: Tierra del Fuego, Argentina. En: Rabassa (ed.): Quaternary of South America and Antarctic Peninsula 4: 291-309.

Rabassa, J.O., A. Coronato, G. Bujalesky, M. Salemme, C. Roig, A. Meglioli, C. Heusser, S. Gordillo, F. Roig, A. Borromei \& M. Quattrocchio 2000. Quaternary of Tierra del Fuego, Southernmost South America: an updated review. International Quaternary 68-71: 217-240.

Rabassa, J., A. Coronato, S. Gordillo, M. Candel \& M. Martinez 2009. Paleoambientes litorales durante la trasgresión marina Holocena en Bahía Lapataia, Canal Beagle, Parque Nacional Tierra del Fuego, Argentina. Revista de la Asociación Geológica Argentina 65: 648-659.

Strelin, J., G. Casassa, G. Rosqvist \& P. Holmlund 2008. Holocene glaciations in the Ema Glacier valley, Monte Sarmiento Massif, Tierra del Fuego. Palaeogeography, Palaeoclimatology, Palaeoecology 260: 299-314. 
Urien, C.M. 1968. Edad de algunas playas elevadas en la Península de Ushuaia y su relación con el ascenso costero postglaciario. Abstracts III Jornadas Geológicas Argentinas 2: 35-41.

Zuschin, M. \& R.J. Stanton 2001. Experimental Measurement of Shell Strength and its Taphonomic Interpretation. Palaios 16, 161-170.
Zuschin, M., M. Stachowitsch \& R.J. Stanton 2003. Patterns and processes of shell fragmentation in modern and ancient marine environments. Earth Science Reviews 63: 33-82. 